\title{
Optical-Feedback Cavity-Enhanced Absorption Spectroscopy with a Quantum Cascade Laser.
}

\author{
G. Maisons ${ }^{2}$, P. Gorrotxategi Carbajo ${ }^{1}$, M. Carras ${ }^{2}$ and D. Romanini ${ }^{1 *}$ \\ ${ }^{1}$ Laboratoire de Spectrométrie Physique, CNRS UMR5588, Université J. Fourier de Grenoble, Saint Martin d'Hères, France \\ 2 Alcatel Thales III-V lab, 1, Av. Augustin Fresnel, Campus Polytechnique, Palaiseau \\ *Corresponding author: daniel.romanini@ujf-grenoble.fr
}

Compiled February 5, 2011

\begin{abstract}
Optical-feedback cavity-enhanced absorption spectroscopy is demonstrated in the mid-IR using a quantum cascade laser (emitting at $4.46 \mu \mathrm{m}$ ). The laser linewidth reduction and frequency locking by selective optical feedback from the resonant cavity field turns out to be particularly important in this spectral range: It allows strong cavity transmission which compensates for low light sensitivity, especially when using room temperature detectors. We obtain a noise equivalent absorption coefficient of $3 \times 10^{-9} \mathrm{~cm}^{-1}$, for $1 \mathrm{~s}$ averaging of spectra composed by 100 independent points. At $4.46 \mu \mathrm{m}$ this yields a detection limit of $35 \mathrm{pptv}$ for $\mathrm{N}_{2} \mathrm{O}$ at 50 mbar, corresponding to $4 \times 10^{7}$ molecules $/ \mathrm{cm}^{3}$, or still to 1 femtomoles in the sample volume.

(C) 2011 Optical Society of America

OCIS codes: $120.6200,280.3420,140.4780,140.5965$,
\end{abstract}

High sensitivity laser absorption spectroscopy, in all its implementations, is widely recognized as a valuable alternative to traditional analytical techniques such as chromatography and mass spectrometry. In many applications it was shown that laser spectroscopy allows more direct, fast, selective, and precise measurement of trace concentrations in gas samples, down to ppbv or pptv levels depending on the strength of the targeted molecular transition. As their performance keeps improving Quantum Cascade Lasers (QCLs) are becoming the source of choice for accessing the strongest molecular transitions in the mid Infra-Red (mid-IR) range. In particular, room temperature single-frequency (DFB) continuously tunable QCLs are now available.

Cavity Enhanced Absorption Spectroscopy (CEAS) and Cavity Ring-Down Spectroscopy (CRDS) usually excel over traditional multipass cells when larger absorption paths are desired. However, in the mid-IR this advantage is thin, since the small throughput of a high finesse cavity is more of a penalty than in the near infrared due to the low detection sensitivity. We present here the first QCL implementation of a high performance CEAS method which exploits Optical Feedback (OF) to achieve optimal injection of the narrow resonances of a high finesse optical cavity [1]. As explained in previous reports of OF-CEAS applications using telecom diode lasers [2-4], OF-CEAS provides rapid but complete, selective and sequential injection of $\mathrm{TEM}_{00}$ cavity modes during a laser scan. Complete injection means that the stationary cavity buildup regime is attained for each $\mathrm{TEM}_{00}$ mode, so that stable cavity output signals are observed with high $\mathrm{S} / \mathrm{N}$ and no averaging is needed to obtain high quality CEAS spectra in a short time (typically 1-2 ms per cavity mode).

A few recent papers describe applications were a QCL is coupled with a high finesse cavity by CEAS or CRDS [5-7]. This was made possible by the advent of contin- uously tuneable CW QCLs, since pulsed QCLs achieve high power during a time comparable to only a few cavity round trips, and in addition during this time its frequency sweeps across several cavity resonances, giving negligible cavity injection. The most impressive performances obtained by coupling a CW QCL to a cavity, are by far those provided by the Off-Axis injection scheme [8], already applied to isotopic ratio measurements aboard aircrafts [9] or to breath analysis in a medical setting [7]. However, despite the high sensitivity and the short measurement times, OA-CEAS is still plagued by low signal levels at cavity output demanding cryogenically cooled, large-area detectors. Another drawback is the large transverse dimension of the high finesse cavity (2 inch diameter mirrors) since OA-CEAS performance depends on the ability to excite very high order transverse cavity modes, whose superposition gives rise to quasi-periodic folded trajectories with Lissajouslike impact patterns optimally filling the mirrors surfaces. The resulting large cavity volumes thus require high sample flows to allow for reasonable response times. Finally, OA-CEAS demands fast laser scanning rates to achieve smoother signals, but at the cost of distorted molecular lineshapes due to the finite cavity response time [9].

OF-CEAS does not suffer from these drawbacks. Its $\mathrm{V}$ shaped cavity attains an internal volume below $20 \mathrm{cc}$. The full injection of well separated longitudinal TEM ${ }_{00}$ cavity modes provides high quality undistorted absorption line profiles on a precisely defined frequency grid (mode spacing for a $50 \mathrm{~cm} \mathrm{V-cavity} \mathrm{is} 150 \mathrm{MHz}$, and definition of mode frequencies is at the $10 \mathrm{kHz}$ level). This feature is to the advantage of high precision measurements as needed e.g. in the determination of isotopic ratios, and it allows using room temperature detectors even in the mid-IR. Indeed the OF-CEAS performance we achieve at $4.5 \mu \mathrm{m}$ when using state of the art mirrors 


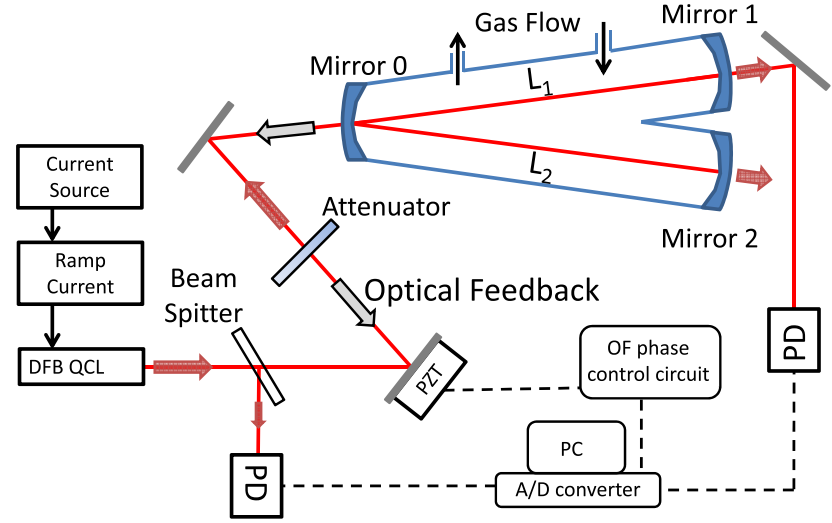

Fig. 1. The V-shaped cavity setup. Part of the resonant intra-cavity field gives frequency selective OF. The PZTmounted steering mirror controls the phase of the $\mathrm{OF}$ field. The laser is mounted on a translation stage to allow wider adjustment of the laser-cavity separation. A beam splitter is used to monitor the laser power.

in this range (99.98\%), is comparable to that we previously obtained in the near-IR when accounting for the higher mirror quality there (99.99\%).

The type of QCL used here (Fig. 1) is detailed in [10]. A metal grating is placed on the top of the laser structure to achieve index modulation with little additional losses. This technology allows reaching room temperature continuous wave monomode emission with $30 \mathrm{~dB}$ of side mode suppression ratio. Around $20^{\circ} \mathrm{C}$ the QCL of our setup has a lasing threshold around $380 \mathrm{~mA}$, and output power of up to $15 \mathrm{~mW}$ for the maximum current of $450 \mathrm{~mA}$. It is thermoregulated by using a Peltier element with a passive air radiator, a thermistance and a commercial diode laser temperature controller. The laser beam is collimated via an aspheric lens with 0.85 numerical aperture. The laser driving current is supplied by a home-made current source as repetitive ramps for periodic laser frequency tuning. A current ramp of $100 \mathrm{~ms}$ from $400 \mathrm{~mA}$ to $450 \mathrm{~mA}$ provides scans over $0.5 \mathrm{~cm}^{-1}$, or about 100 cavity modes. A special effort was needed for adapting an electronic card originally developed for diode lasers, in order to keep the current noise at a sufficiently low level.

The photodiodes (PD) are $(\mathrm{HgCdZn}) \mathrm{Te}$ structures by VIGO with a detectivity of $6 \times 10^{10} \mathrm{~cm} \sqrt{\mathrm{Hz}} / \mathrm{W}$ for the $\mathrm{PD}$ measuring the cavity transmission and $2 \times 10^{10} \mathrm{~cm} \sqrt{\mathrm{Hz}} / \mathrm{W}$ for the reference signal PD. Their bandpass is $1 \mathrm{MHz}$. As usual in OF-CEAS, an attenuator controls the fraction of returning radiation (feedback rate): in this setup, we use two microscope glass slabs ( $1 \mathrm{~mm}$ thick) giving about $21 \%$ transmission each. Also, the OF phase is finely controlled by sub-wavelength automatic displacements of the PZT mounted mirror while the QCL is mounted on a translation stage for rough adjustment of the laser-cavity distance [1]. A sealed glass

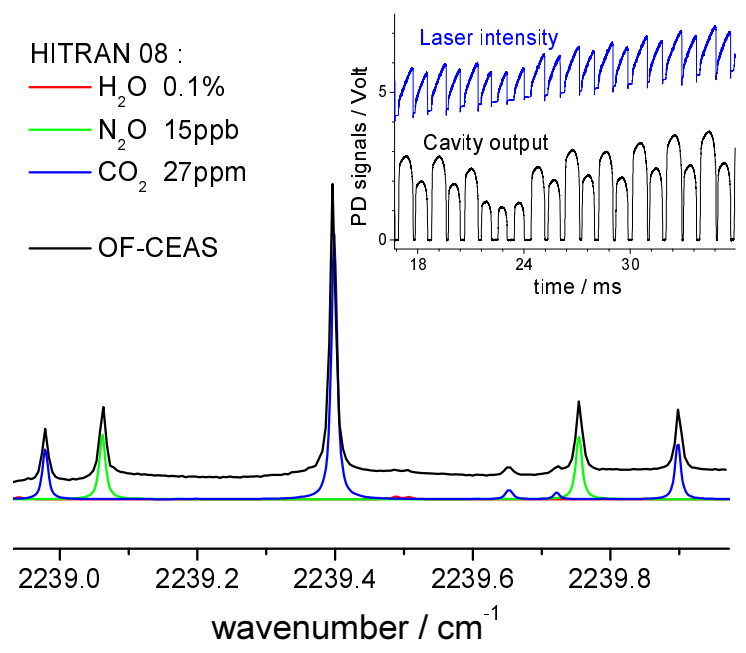

Fig. 2. OF-CEAS spectra of diluted air, 1:25 in dry $\mathrm{N}_{2}$ (black line), together with a HITRAN simulation (colours). Baseline offset in the OF-CEAS spectrum corresponds to the empty cavity losses. The inset displays a section of the photodetectors signals, whose ratio gives the cavity transmission: To be noticed the large laser power increase in presence of resonant cavity feedback.

cell, including the cavity mirrors, enables using low pressure samples (measured by a capacitive gauge) with a controlled flow from parallel mounted Mass Flow Controllers (MFC). High reflectivity dielectric mirrors are by Lohnstar Optics. They are made on superpolished ZnSe substrates and their stopband goes from about 4.3 to $4.8 \mu \mathrm{m}$. From our ringdown measurements reflectivity is $99.98 \%$ at $4.47 \mu \mathrm{m}$. We also directly measured a mirror transmission of $110 \pm 20 \mathrm{ppm}$, which implies mirror coating losses (absorption plus scattering) of about $90 \mathrm{ppm}$.

A 16-bit A/D card connected to a PC records the photodetector signals. In the inset of Fig. 2 we display examples of these signals (a span over about 20 cavity modes) which yield the CEAS mode-by-mode spectra as described previously [2]. A ring-down is produced at the end of each scan by a short programmed interruption of the laser injection current, which allows to calibrate the absorption scale of the CEAS spectrum [4].

A temperature ramp between 18.5 and $22.5^{\circ} \mathrm{C}$ tunes our QCL in the range from $2238.85 \mathrm{~cm}^{-1}$ to $2240 \mathrm{~cm}^{-1}$. Fig. 2 shows the OF-CEAS spectrum for diluted ambient air (about 1:25 in dry nitrogen) at a total pressure of 50 mbar, reconstructed from several current scans taken as the laser is temperature-tuned over this range. A HITRAN simulation for close-to-standard air composition and accounting for the dilution is also shown. Carbon dioxide $\mathrm{CO}_{2}$ and nitrous oxide $\mathrm{N}_{2} \mathrm{O}$, are clearly the main absorbing species, while water vapor lines are barely visible (after dilution). In order to check reproducibility and linearity, integrated line absorption and pressure broad- 


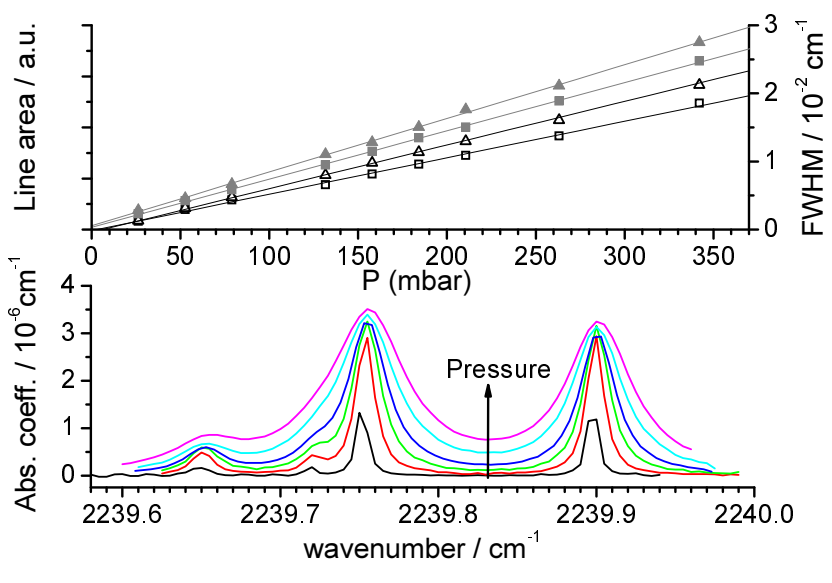

Fig. 3. Bottom graph, OF-CEAS spectra displaying linewidth broadening of $\mathrm{CO}_{2}(2239.898,2239.722$, and $\left.2239.652 \mathrm{~cm}^{-1}\right)$ and $\mathrm{N}_{2} \mathrm{O}\left(2239.754 \mathrm{~cm}^{-1}\right)$ lines as a function of total pressure for a constant air dilution factor (1:10 in dry nitrogen) at total pressures of 26,52 , 131, 185, 263 and 340 mbar. Baseline of spectra (from line profiles fit) has been subtracted. On top, linear dependence of the Lorentzian broadening (grey) and the line surface (black) for $\mathrm{CO}_{2}$ (squares) and $\mathrm{N}_{2} \mathrm{O}$ (triangles) with increasing total pressure.

ening measurements are carried out for these species. As the absorption lines are fit to Voigt profiles, a fifth order polynomial is included to adjust for a slow baseline modulation due to etaloning in the mirror substrates (unwedged).

Line intensities are determined for different dilutions of ambient air in dry nitrogen at a constant pressure of 50 mbar, while linewidth broadening coefficients are obtained for different total pressures at a constant dilution factor. A linear enhancement of $\mathrm{N}_{2} \mathrm{O}$ and $\mathrm{CO}_{2}$ concentrations is indeed observed to within experimental errors when increasing the flow rate of ambient air in dry nitrogen to cover the dilution range from 1 to $20 \%$. Fig. 3 resumes more interesting results as a function of pressure. The bottom plot shows spectra taken for a total pressure varying from 26 to 350 mbar where Lorentzian broadening starts from below the Doppler broadening and then becomes dominant. The graph at the top illustrates the excellent linear dependence of the Lorentzian widths and the surfaces of the absorption lines with increasing pressure. These measurements also illustrate that below 50 mbar the Doppler broadening starts becoming dominant for these small molecules. This pressure thus satisfies the need for narrow and well separated absorption lines while still maintaining line peak values almost at their max value.

The noise in the recorded spectra is estimated from the residuals of the fit to all the $\mathrm{NO}_{2}$ and $\mathrm{CO}_{2}$ lines present in the spectrum of Fig. 3. The noise equivalent absorption is $3 \times 10^{-9} \mathrm{~cm}^{-1}$ for $1 \mathrm{~s}$ averaging over laser scans, which results in an averaged spectrum of about 100 independent points, one per cavity mode. From this baseline noise, the detection limit for $\mathrm{N}_{2} \mathrm{O}$ and $\mathrm{CO}_{2}$ are estimated to be around $35 \mathrm{pptv}$ and $15 \mathrm{ppbv}$, respectively. Equivalently, the rms noise on the concentration trace of these molecules from spectral fitting at successive times (at a fixed 50 mbar optimal pressure, and with sufficient dilution) is also found to be consistent with these detection limits. For $\mathrm{N}_{2} \mathrm{O}$ at 50 mbar, we obtain a corresponding smallest detectable number density of $4 \times 10^{7}$ molecules $/ \mathrm{cm}^{3}$. Considering the sample volume this may also be cast to $10^{9}$ molecules or equivalently $\sim 1$ femtomoles.

With this work we demonstrate for the first time the high potential of OF-CEAS coupled with CW QCL light sources for gas analysis or high sensitivity precision molecular spectroscopy. The detection limit $\left(3 \times 10^{-9} / \mathrm{cm} \sqrt{\mathrm{Hz}}\right.$ for an averaged spectrum of 100 points $)$ is close to that obtained for DFB diode lasers in the near IR range when scaled for the cavity ring-down time (e.g. a factor 3 larger in ref [2] where a detection limit of $10^{-9} / \mathrm{cm}$ is obtained with $1 \mathrm{~s}$ averaging). Compared with DFB diode lasers, QCLs seem to respond more markedly to the cavity feedback (in similar conditions of feedback levels). We observe power enhancements of 15-20\% (10 times more than for a DFB diode laser), which may be shown to be in good agreement with theoretical predictions based on the model of P. Laurent [11] implemented with QCL parameters.

We acknowledge financing by the French Agence $\mathrm{Na}$ tional de la Recherche (APOLINR project).

\section{References}

1. J. Morville, S. Kassi, M. Chenevier, and D. Romanini, "Fast, low-noise, mode-by-mode, cavity-enhanced absorption spectroscopy by diode-laser self-locking," Appl. Phys. B 80, 1027-1038 (2005).

2. D. Romanini, M. Chenevier, S. Kassi, M. Schmidt, C. Valant, M. Ramonet, J. Lopez, and H.-J. Jost, "Optical-feedback cavity-enhanced absorption: A compact spectrometer for real-time measurement of atmospheric methane," Appl. Phys. B 83, 659-667 (2006).

3. S. Kassi, M. Chenevier, L. Gianfrani, A. Salhi, Y. Rouillard, A. Ouvrard, and D. Romanini, "Looking into the volcano with a Mid-IR DFB diode laser and Cavity Enhanced Absorption Spectroscopy," Optics Express 14, 11,442-11,452 (2006).

4. E. R. T. Kerstel, R. Q. Iannone, M. Chenevier, S. Kassi, H.-J. Jost, and D. Romanini, "A water isotope $\left({ }^{2} \mathrm{H},{ }^{17} \mathrm{O}\right.$, and ${ }^{18} \mathrm{O}$ ) spectrometer based on optical feedback cavityenhanced absorption for in situ airborne applications," Appl. Phys. B 85, 397-406 (2006).

5. A. A. Kosterev, A. L. Malinovsky, F. K. Tittel, C. Gmachl, F. Capasso, D. L. Sivco, J. N. Baillargeon, A. L. Hutchinson, and A. Y. Cho, "Cavity ringdown spectroscopic detection of nitric oxide with a continuouswave quantum-cascade laser," Appl. Opt. 40, 5522-5529 (2001).

6. A. A. Kosterev and F. K. Tittel, "Chemical Sensors 
Based on Quantum Cascade Lasers," IEEE J. Quantum Electron. 38, 582-591 (2002).

7. K. R. Parameswaran, D. I. Rosen, M. G. Allen, A. M. Ganz, and T. H. Risby, "Off-axis integrated cavity output spectroscopy with a mid-infrared interband cascade laser for real-time breath ethane measurements," Appl. Opt. 48, B73-B79 (2009).

8. J. B. Paul, L. Lapson, and J. G. Anderson, "Ultrasensitive absorption spectroscopy with a high-finesse optical cavity and off-axis alignment," Appl. Opt. 40, 49044910 (2001).

9. E. J. Moyer1, D. S. Sayres, G. S. Engel, J. M. S. Clair, F. N. Keutsch, N. T. Allen, J. H. Kroll, and J. G. Anderson, "Design considerations in high-sensitivity off-axis integrated cavity output spectroscopy," Appl. Phys. B 92, 467-474 (2008).

10. M. Carras, G. Maisons, B. Simozrag, M. Garcia, O. Parillaud, and X. Marcadet, "Room-temperature continouswave metal grating distributed feedback quantum cascade lasers," Appl. Phys. Lett. 96, 161,105 (2010).

11. P. Laurent, A. Clairon, and C. Breant, "Frequency Noise Analysis of Optical Self-Locked Diodes Lasers," IEEE J. Quantum Electron. 25, 1131-1142 (1989). 